\title{
СОВРЕМЕННЫЕ ТЕНДЕНЦИИ В ЗАРУБЕЖНЫХ ИССЛЕДОВАНИЯХ КОГНИТИВНЫХ ИСКАЖЕНИЙ В ПРОЦЕССАХ ПРИНЯТИЯ РЕШЕНИЙ
}

\section{Н.И. ЛОГИНОВ ${ }^{\mathrm{a}, \mathrm{b}}$, А.С. АЛЕКСАНДРОВА}

\author{
${ }^{a}$ Российская академия народного хозяйства и государственной службы при Президенте \\ Российской Федеращии, 119571, Россия, Москва, просп. Вернадского, д. 82-84 \\ ${ }^{b}$ Национальный исследовательский университет «Высшая школа экономики», 101000, Россия, \\ Москва, ул. Мясницкал, д. 20
}

\begin{abstract}
Резюме
Когнитивные искажения, возникающие в процессе мышления и принятия решений, становятся все более обсуждаемой темой, вызывают споры и дебаты относительно психологических механизмов и теорий, лежащих в их основе. Вопросы, касающиеся рациональности принятия решений и отклонений от рациональности, возникают во многих областях жизнедеятельности и особо остро интересуют маркетологов, политиков, дизайнеров и людей, на которых лежит большая ответственность в принятии важных решений. В настоящее время в исследованиях когнитивных искажений в этих областях появляется все больше эмпирических доказательств таких отклонений, но теоретическая модель и лежащие в ее основе механизмы продолжают уточняться. Данная статья посвящена обзору наиболее перспективных тенденций в исследованиях когнитивных искажений, ориентированных на прояснение устройства психологических механизмов, лежащих в их основе. Одним из аспектов в современных трендах двухпроцессных теорий является изучение индивидуальных различий в процессе использования двух систем и, как следствие, склонности к когнитивным искажениям. Другим направлением является практическое, которое, опираясь на данные эффекты, использует их для определенных целей. В цифровой среде основанные на когнитивных искажениях приемы активно применяются дизайнерами интерфейсов. В статье приводятся приемы подталкиваний и приемы внедрения знаний и прививания компетенций, при которых с помощью изменений цифровой среды выбор пользователя может быть направлен в сторону определенной альтернативы, при этом фактического выбора его не лишают. Использование данных приемов также вызывает критические замечания по поводу их уместности и действительной эффективности, что требует дополнительных теоретических и практических изысканий в данной области.
\end{abstract}

Ключевые слова: принятие решений, двухпроцессные теории, теории двух систем, когнитивные искажения, наджинг, бустинг.

\section{Введение}

Одна из наиболее известных и масштабных исследовательских программ в области психологии принятия решений связана с работами Д. Канемана и

Исследование осуществлено в рамках Программы фундаментальных исследований НИУ ВШЭ в 2019 г. 
A. Тверски (Tversky, Kahneman, 1974; Kahneman et al., 1982) и посвящена изучению эвристик и когнитивных искажений. Понятие когнитивных искажений относится к широкому спектру систематических отклонений от рациональности в процессах принятия решений, основной источник которых коренится в самом устройстве познавательных процессов. Под эвристиками же в данном научном направлении понимаются психологические механизмы, позволяющие быстро принимать решения на основе ограниченного количества информации и зачастую для этого жертвующие точностью, что и приводит к целому ряду систематических ошибок (Gigerenzer, Gaissmaier, 2011). Данная линия исследований уже продемонстрировала колоссальное количество эмпирических свидетельств в пользу существования разнообразных когнитивных искажений и эвристик, лежащих в их основе (Stanovich, Toplak, West, 2008).

Однако благодаря активной популяризации поведенческой экономики и когнитивной психологии принятия решений к когнитивным искажениям стали обращаться в публичных дискуссиях для объяснения политических, религиозных и - шире - мировоззренческих позиций оппонента, что бумерангом отразилось на качестве теорий в этой области (Ariely, 2008; Kahneman, 2011). В результате это привело к постепенному размыванию границ понятия когнитивных искажений, когда к ним начали относить иллюзии зрительного восприятия и ошибки памяти, а также к стагнации в развитии теоретических представлений о природе когнитивных искажений, остающихся либо на уровне теорий двух систем, либо по поводу и без вводящих все новые сущности для объяснения - эвристики. Помимо этого, прирост исследований в этой области происходит за счет появления колоссального количества попыток найти когнитивные искажения в различных прикладных областях и справиться с ними (например: Burmeister, Schade, 2007; Blumenthal-Barby, Krieger, 2015; Zhang, Cueto, 2017).

Данная статья посвящена обзору наиболее перспективных тенденций в исследованиях когнитивных искажений, ориентированных на прояснение устройства психологических механизмов, лежащих в их основе.

\section{Двухпроцессные теории принятия решений}

В первую очередь современные тенденции в исследованиях этой области связаны с пересмотром некоторых классических теоретических построений, опирающихся на теорию двух систем мышления Д. Канемана, а также в целом на большой класс теорий, различающих два класса процессов обработки информации по разным, но, как кажется авторам, семантически схожим основаниям (Evans, Stanovich, 2013). Двухпроцессные теории в исследованиях мышления были определены в работах У. Джеймса (James, 1890) и по настоящее время являются темой для обширных дебатов и рассуждений о природе и взаимодействии двух составляющих процессов. При условном разделении процессов первый тип рассматривают как интуитивный или эвристический, а второй - как логический или аналитический. Некоторые авторы дополнительно 
предполагают, что в основе этих двух типов обработки информации лежат две эволюционно различные системы мозга (Stanovich, 1986; Epstein, 1994; Evans, Over, 1996; Reber, 1993). Такие теории зачастую опираются на идею о том, что существует эволюционно более древняя и присущая всем животным форма мышления, и ей противопоставляется недавно развитая и уникальная человеческая система мышления.

Так или иначе, дебаты относительно двухпроцессных теорий в области принятия решений составляют одну из ключевых линий в развитии современных научных представлений. Наиболее строгая и полная попытка описания двухпроцессных моделей, а также проблем и возможных решений, с ними связанных, была предпринята в работе Дж. Эванса и К. Становича (Evans, Stanovich, 2013). Они предложили различать сущностные характеристики этих двух типов процессов и то, с чем эти характеристики могут коррелировать. К сущностным характеристикам были отнесены нагрузка на рабочую память (необходимая для процессов второго типа и необязательная для процессов первого), а также автономность (принудительный характер протекания процессов первого типа в присутствии соответствующих триггеров и отсутствие такой особенности у процессов второго типа). Остальные же характеристики были отнесены к коррелятам. Отдельное внимание авторы уделили разбору критических замечаний в адрес двухпроцессных моделей, указав на то, что они друг от друга отличаются довольно сильно и критиковать их в целом смысла нет.

К наиболее часто встречающимся общим критическим замечаниям в адрес двухпроцессных моделей авторы отнесли:

а) отсутствие строгих определений (Smith, DeCoster, 2000; Evans, 2003; Smith, Collins, 2009);

б) недостаток согласованности и последовательности в описании характеристик двух типов процессов (Keren, Schul, 2009);

в) наличие скорее количественных, чем качественных различий между двумя типами процессов (например, скорость переработки информации) (Newstead, 2000; Osman, 2004);

г) возможность объяснения данных, полученных в ходе экспериментальных исследований, функционированием лишь одного процесса, а не двух отдельных (Gigerenzer, Regier, 1996; Osman, 2004; Keren, Schul, 2009);

д) недостатки экспериментальных свидетельств, подтверждающих двухпроцессные теории (Kruglanski, Gigerenzer, 2011).

Однако легитимность этих критических замечаний продолжает активно обсуждаться (Thompson, Newman, 2018; Melnikoff, Bargh, 2018; Bago, De Neys, 2019; De Neys, Pennycook, 2019), авторы оспаривают аргументы друг друга. В связи с этим на данный момент затруднительно определить статус двухпроцессных и двухсистемных моделей в данной исследовательской области. Тем не менее основной прогресс ожидается именно в связи с ответом на вопрос, является ли этот вид моделей достаточно эвристически ценным для объяснения полученных фактов в исследованиях процессов принятия решений, вынесения суждений и умозаключений. 
Уже сейчас можно отметить небольшой прогресс в этом направлении. В частности, Эванс и Станович (Evans, Stanovich, 2013) предложили различать два типа двухпроцессных моделей и проверять их отдельно:

а) дефолтно-интервенционистские, предполагающие, что процессы первого типа работают в режиме «по умолчанию», а процессы второго типа лишь вмешиваются при необходимости;

б) конкурентно-параллельные, опирающиеся на представления о том, что процессы первого и второго типа соревнуются друг с другом в режиме реального времени.

В современных исследованиях в основном предпочтение отдается проверкам дефолтно-интервенционистских моделей (Furley et al., 2015; Bago, De Neys, 2019), к которым относится и теория двух систем мышления Канемана.

При этом классическое представление о том, что именно Система 1 ответственна за когнитивные искажения и систематические ошибки, а Система 2 вынуждена их отслеживать и исправлять, периодически сталкивается с эмпирическим опровержением (Goldstein, Gigerenzer, 2011; Raab, Gigerenzer, 2015). Наиболее активным критиком этой идеи выступает Г. Гигеренцер, автор концепции экологической рациональности, согласно которой эвристики, на которые по мнению Канемана опирается Система 1, адаптивны по своей природе и помогают нам точно и быстро принимать столь необходимые в повседневной жизни дорефлексивные решения (Gigerenzer, Gaissmaier, 2011).

Еще одним важным направлением пересмотра представлений о природе когнитивных искажений являются исследования индивидуальных различий в склонности к когнитивным искажениям. Согласно классической точке зрения, представленной теорией двух систем Д. Канемана, возникновение когнитивных искажений связано с работой Системы 1, а возможности совладать с ними предоставляет Система 2. Однако большое количество эмпирических исследований демонстрирует, что люди могут не быть подвержены конкретным когнитивным искажениям, даже если не предпринимают каких-либо усилий для того, чтобы отслеживать их возникновение и своевременно их элиминировать (Stanovich, West, 2000).

Современные исследования в этом направлении активно ищут диспозициональные предикторы, которые определяют то, в какой степени и с какой вероятностью конкретный человек будет склонен к эффекту фрейминга или ошибке подтверждения (West, Toplak, Stanovich, 2008).

\section{Когнитивные искажения в цифровой среде}

Обсуждавшиеся выше тренды были связаны преимущественно с развитием фундаментальных исследований и с некоторым запросом на уточнение теоретических моделей. Однако, помимо этих тенденций, можно выделить также и те, что связаны в основном с запросом со стороны практики и развития современных цифровых технологий. В частности, дизайнеры, разработчики и проектировщики цифровой среды сталкиваются с необходимостью учитывать особенности восприятия, внимания и мышления людей для повышения 
эффективности взаимодействия с сайтом, удовлетворенности пользователей, увеличения заинтересованности и времени, проводимого на сайте (Lee, Koubek, 2010; Lindgaard et al., 2006). В связи с развитием исследований в области поведенческой экономики и когнитивной психологии принятия решений дизайнеры обнаружили свою заинтересованность в изменениях цифровой среды, в которой происходит выбор, для направления и побуждения лиц, принимающих решение, к определенной альтернативе, при этом оставляя перед ними возможность выбора (Halpern, 2015). Данные стратегии для достижения определенных целей называются подталкиванием, или наджингом (nudging) (Thaler, Sunstein, 2008).

Подход с использованием подталкиваний основан на теории двух систем в классическом варианте. Попытки изменить поведение с помощью подталкиваний могут либо усиливать вовлечение Системы 2, либо использовать недостатки Системы 1 в виде систематических когнитивных искажений. С опорой на теории двух систем и исследования эвристик в принятии решений развивались вопросы о том, как внешняя среда и контекст выбора влияют на реализуемый выбор (Lichtenstein, Slovic, 2006). К. Санстейн (2019) выделил основные типы подталкивания, которые уже используются в цифровом пространстве для изменения тенденции при выборе:

1) значения по умолчанию, когда более предпочтительный вариант выбран автоматически и требуются усилия, чтобы выбрать иной вариант;

2) упрощение сложных документов до ключевых моментов;

3) напоминания об оплатах, важных событиях, специальных предложениях;

4) вопросы по поводу намерений;

5) привлечение мнений других людей и апелляции к социальным нормам;

6) повышение простоты и удобства для выбора определенных альтернатив;

7) приведение статистических данных в понятной форме;

8) предупреждения о последствиях;

9) предоставление информации о предыдущих решениях и их последствиях.

Подталкивания по своему замыслу как приемы основаны на когнитивных искажениях и стилистических особенностях принятия решений. Чаще всего в работах, посвященных подталкиваниям, можно встретить следующие когнитивные искажения:

- Эффект фрейминга (Tversky, Kahneman, 1986), который заключается в том, что результат выбора зависит от представления выбора как вопроса о выигрыше или о проигрыше (Liu et al., 2019).

- Избегание потерь (Tversky, Kahneman, 1991), которое заключается в том, что потери кажутся субъективно бо́льшими и менее предпочтительными, чем объективно равные им по ценности выгоды. На сайтах, рекламирующих предлагаемый товар, используется прием формулирования информации в терминах угрозы потерять выгодное предложение. Например, изображен счетчик времени, указывающий на длительность краткого периода и показывающий, как истекает время предложения. Другой пример - информация о спросе на 
товар, о количестве купленных товаров за конкретный промежуток времени, так как повышенный спрос и ограничения по времени формулируют задачу в терминах потерь, которые может нести покупатель, если не воспользуется предложением как можно быстрее (Stryja, Satzger, 2019).

- Эффект обладания, заключающийся в том, что человек больше ценит те вещи, которыми уже владеет, а не те, которыми может овладеть (Kahneman et al., 1990). Предложение бесплатного пробного периода пользования приложением или услугами располагает к большей готовности продлить платную услугу, так как пользователь ценит уже имеющийся товар больше, чем потенциальный (Fritze et al., 2019).

Другим приемом, используемым для вмешательства в процесс выбора, является бустинг (boosting) (Grüne-Yanoff, Hertwig, 2016). Целью бустинга является повышение способностей людей более рационально делать свой собственный выбор. Основное внимание уделяется вмешательствам, которые облегчают людям их собственную деятельность, развивая существующие компетенции или прививая новые. Примером может служить развитие способности понимать статистическую информацию о состоянии здоровья или повышение финансовой грамотности. При этом у человека всегда есть выбор использовать новые навыки и знания для дальнейшего выбора или нет. Успешное применение бустинга было осуществлено в отношении когнитивного искажения пренебрежения базовым уровнем, при котором люди склонны игнорировать общую информацию о частоте некоторых событий и находить специфическую информацию о нем. В случае, когда решение принималось на основе вероятностей наступления некоторого события, люди совершали больше ошибок, чем в случае, когда с помощью бустинга людям давали информацию, выраженную в частотах, а не вероятностях (Hoffrage et al., 2000), и впоследствии предоставляли инструменты (Sedlmeier, Gigerenzer, 2001), с помощью которых можно было самостоятельно переводить вероятности в частоты для принятия решения.

Таким образом, наджинг и бустинг - это способы воздействия на процесс принятия решений, уже активно применяемые в цифровой среде. Наджинг в большей степени нацелен на изменение среды выбора, а бустинг - способностей и знания лиц, принимающих решения. Их внедрение требует меньших затрат по сравнению с естественной средой выбора в различных сфера жизни, однако эффективность требует дополнительной проверки в последующих исследованиях.

\section{Заключение}

Современный статус двухпроцессных теорий в области принятия решений остается неоднозначным и требует дальнейших прояснений с учетом большого количества новых фактов об индивидуальных различиях в когнитивных искажениях и развития сопутствующих психометрических инструментов. Отдельно стоит отметить необходимость разработки и/или адаптации психометрических инструментов на русском языке. Отсутствие явного прогресса в 
направлении развития процедур измерения склонности к когнитивным искажениям сильно контрастирует с большим количеством попыток использовать наработки этой области в различных прикладных сферах с целью оптимизации процессов принятия решений. Данное обстоятельство только повышает актуальность проблематики когнитивных искажений и запрос на развернутые, в том числе, отечественные исследовательские программы.

Ссылки на зарубежные источники см. в разделе References после англоязычного блока.

Логинов Никита Иванович - доцент, кафедра общей психологии; научный сотрудник, Лаборатория когнитивных исследований, ИОН РАНХиГС; научный сотрудник, Научно-учебная лаборатория когнитивной психологии пользователя цифровых интерфейсов, Национальный исследовательский университет «Высшая школа экономики», кандидат психологических наук.

Сфера научных интересов: инсайт, принятие решений, когнитивные искажения, ментальные модели, воплощенное познание.

Контакты: lognikita@yandex.ru

Александрова Анастасия Игоревна - студент магистратуры; стажер-исследователь, Научно-учебная лаборатория когнитивной психологии пользователя цифровых интерфейсов, Национальный исследовательский университет «Высшая школа экономики».

Сфера научных интересов: решение задач, принятие решений, когнитивные искажения, дизайн интерфейсов.

Контакты: 1aialeksandrova@gmail.com

\title{
Current Trends in International Research on Cognitive Biases in Decision-Making Processes
}

\author{
N.I. Loginova,b A.I. Aleksandrova ${ }^{\mathrm{b}}$ \\ ${ }^{a}$ Russian Presidential Academy of National Economy and Public Administration (RANEPA), 82-84 \\ Prospect Vernadskogo, Moscow, 119571, Russian Federation \\ ${ }^{b}$ National Research University Higher School of Economics, 20 Myasnitskaya Str., Moscow, 101000, \\ Russian Federation
}

\begin{abstract}
Cognitive biases emerging in judgments and decision-making become an increasingly discussed topic and cause controversy and debate regarding the psychological mechanisms and underlying theories. Questions regarding rational decision-making and deviations from rationality arise in many areas of life and are of a particular interest to marketers, politicians, designers and people who bear high responsibility in making important decisions. Currently, more empirical evidence for these deviations appears in studies of cognitive biases in the areas, but also the theoretical model and the basis of the underlying mechanisms continue to be refined. This article is devoted to the latest trends regarding two-process theories of thinking and the subsequent
\end{abstract}


criticism. The development of these theories and their varieties is described in detail and the main trends based on these theories are highlighted. One of the aspects in current trends of the two-process theories is the study of individual differences on the use of the two systems and the level of tendency to cognitive biases. Another direction is the practical component, which is based on these effects. In a digital environment, cognitive-biases-based techniques are actively used by interface designers. The article presents nudging and boosting methods in which with the help of changes in the digital environment the user's choice can be directed towards a certain alternative, while the actual choice remains. These techniques also have critical comments on the appropriateness of the application and their actual effectiveness, which requires additional theoretical and practical research in this area.

Keywords: decision-making, dual process theories, two system theories, cognitive biases, nudging, boosting.

\section{References}

Ariely, D. (2008). Predictably irrational. New York: Harper Audio.

Bago, B., \& De Neys, W. (2019). The smart System 1: Evidence for the intuitive nature of correct responding on the bat-and-ball problem. Thinking and Reasoning, 25(3), 257-299.

Blumenthal-Barby, J. S., \& Krieger, H. (2015). Cognitive biases and heuristics in medical decision making: a critical review using a systematic search strategy. Medical Decision Making, 35(4), 539 557.

Burmeister, K., \& Schade, C. (2007). Are entrepreneurs' decisions more biased? An experimental investigation of the susceptibility to status quo bias. Journal of Business Venturing, 22(3), 340-362.

De Neys, W., \& Pennycook, G. (2019). Logic, fast and slow: Advances in dual-process theorizing. Current Directions in Psychological Science, 28(5), 503-509.

Epstein, S. (1994). "Integration of the cognitive and the psychodynamic unconscious": Comment. American Psychologist, 50(9), 799-800.

Evans, J. S. B. (2003). In two minds: dual-process accounts of reasoning. Trends in Cognitive Sciences, 7(10), 454-459.

Evans, J. S. B. T., \& Over, D. E. (1996). Rationality and reasoning. Oxford, UK: Psychology/Erlbaum (UK) Taylor \& Francis.

Evans, J. S. B. T., \& Stanovich, K. E. (2013). Dual-process theories of higher cognition: Advancing the debate. Perspectives on Psychological Science, 8(3), 223-241.

Fritze, M. P., Eisingerich, A. B., \& Benkenstein, M. (2019). Digital transformation and possession attachment: examining the endowment effect for consumers' relationships with hedonic and utilitarian digital service technologies. Electronic Commerce Research, 19(2), 311-337.

Furley, P., Schweizer, G., \& Bertrams, A. (2015). The two modes of an athlete: dual-process theories in the field of sport. International Review of Sport and Exercise Psychology, 8(1), 106-124.

Gigerenzer, G., \& Gaissmaier, W. (2011). Heuristic decision making. Annual Review of Psychology, 62, 451-482.

Gigerenzer, G., \& Regier, T. (1996). How do we tell an association from a rule? Comment on Sloman (1996). Psychological Bulletin, 119(1), 23-26. 
Goldstein, D. G., \& Gigerenzer, G. (2011). The beauty of simple models: Themes in recognition heuristic research.Judgment and Decision Making, 6(5), 392-395.

Grüne-Yanoff, T., \& Hertwig, R. (2016). Nudge versus boost: How coherent are policy and theory? Minds and Machines, 26(1-2), 149-183.

Halpern, D. (2015). Inside the nudge unit: How small changes can make a big difference. Random House.

Hoffrage, U., Lindsey, S., Hertwig, R., \& Gigerenzer, G. (2000). Medicine. Communicating statistical information. Science, 290(5500), 2261-2262.

James, W. (1890). The principles of psychology. New York: Henry Holt and Company.

Kahneman, D. (2011). Thinking, fast and slow. Macmillan.

Kahneman, D., Knetsch, J. L., \& Thaler, R. H. (1990). Experimental tests of the endowment effect and the Coase theorem. Journal of Political Economy, 98(6), 1325-1348.

Kahneman, D., Slovic, P., \& Tversky, A. (Eds.). (1982).Judgment under uncertainty: Heuristics and biases. Cambridge University Press.

Keren, G., \& Schul, Y. (2009). Two is not always better than one: A critical evaluation of two-system theories. Perspectives on Psychological Science, 4(6), 533-550.

Kruglanski, A. W., \& Gigerenzer, G. (2011). Intuitive and deliberate judgments are based on common principles. Psychological Revieze, 118(1), 97-109.

Lee, S., \& Koubek, R. (2010). Understanding user preferences based on usability and aesthetics before and after actual use. Interacting with Computers, 22, 530-543.

Lichtenstein, S., \& Slovic, P. (2006). The construction of preference: An overview. In S. Lichtenstein \& P. Slovic (Eds.), The construction of preference (pp. 1-40). Cambridge University Press.

Lindgaard, G., Fernandes, G., Dudek, C., \& Brown, J. (2006). Attention web designers: You have 50 milliseconds to make a good first impression! Behaviour and Information Technology, 25(2), 115-126.

Liu, J., Lee, B. G., McLeod, D. M., \& Choung, H. (2019). Effects of frame repetition through cues in the online environment. Mass Communication and Society, 22(4), 447-465.

Melnikoff, D. E., \& Bargh, J. A. (2018). The mythical number two. Trends in Cognitive Sciences, 22(4), 280-293.

Newstead, S. E. (2000). Are there two different types of thinking? Behavioral and Brain Sciences, 23(5), 690-691.

Osman, M. (2004). An evaluation of dual-process theories of reasoning. Psychonomic Bulletin and Revier, 11(6), 988-1010.

Raab, M., \& Gigerenzer, G. (2015). The power of simplicity: a fast-and-frugal heuristics approach to performance science. Frontiers in Psychology, 6, Art. N 1672.

Reber, A. S. (1993). Implicit learning and tacit knowledge: an essay on the cognitive unconscious. New York: Oxford University Press.

Sedlmeier, P., \& Gigerenzer, G. (2001). Teaching Bayesian reasoning in less than two hours. Journal of Experimental Psychology: General, 130(3), 380-400.

Smith, E. R., \& Collins, E. C. (2009). Dual-process models: A social psychological perspective. In J. St. B. T. Evans \& K. Frankish (Eds.), In two minds: Dual processes and beyond (pp. 197-216). Oxford: Oxford University Press.

Smith, E. R., \& DeCoster, J. (2000). Dual-process models in social and cognitive psychology: Conceptual integration and links to underlying memory systems. Personality and Social Psychology Revier, 4(2), 108-131.

Stanovich, K. E. (1986). How to think straight about psychology. New York: HarperCollins. 
Stanovich, K. E., Toplak, M. E., \& West, R. F. (2008). The development of rational thought: A taxonomy of heuristics and biases. Advances in Child Development and Behavior, 36, 251-285.

Stanovich, K. E., \& West, R. F. (2000). Individual differences in reasoning: Implications for the rationality debate? Behavioral and Brain Sciences, 23(5), 645-665.

Stryja, C., \& Satzger, G. (2019). Digital nudging to overcome cognitive resistance in innovation adoption decisions. The Service Industries Journal, 39(15-16), 1123-1139.

Thaler, R. H., \& Sunstein, C. R. (2008). Nudge: Improving decisions about health, wealth, and happiness. New Haven, CT: Yale University Press.

Thompson, V. A., \& Newman, I. R. (2018). Logical intuitions and other conundra for dual process theories. In W. De Neys (Ed.), Current issues in thinking and reasoning. Dual process theory 2.0 (pp. 121-136). Routledge/Taylor \& Francis Group.

Tversky, A., \& Kahneman, D. (1974). Judgment under uncertainty: Heuristics and biases. Science, 185(4157), 1124-1131.

Tversky, A., \& Kahneman, D. (1986). Rational choice and the framing of decisions. The Journal of Business, 59(4), S251-S278.

Tversky, A., \& Kahneman, D. (1991). Loss aversion in riskless choice: A reference-dependent model. The Quarterly Journal of Economics, 106(4), 1039-1061.

West, R. F., Toplak, M. E., \& Stanovich, K. E. (2008). Heuristics and biases as measures of critical thinking: Associations with cognitive ability and thinking dispositions. Journal of Educational Psychology, 100(4), 930-941.

Zhang, S. X., \& Cueto, J. (2017). The study of bias in entrepreneurship. Entrepreneurship Theory and Practice, 41(3), 419-454.

Nikita I. Loginov - Associate Professor, Faculty of Psychology; Research Fellow, Laboratory of cognitive research, ISS RANEPA; Research Fellow, Cognitive Psychology of Digital Interface Users, National Research University Higher School of Economics, PhD in Psychology.

Research Area: insight, decision making, cognitive biases, mental models, embodied cognition.

E-mail: lognikita@yandex.ru

Anastasia I. Aleksandrova - HSE Master student, Research Assistant, Cognitive Psychology of Digital Interface Users, National Research University Higher School of Economics.

Research Area: problem solving, decision making, cognitive biases, interface design.

E-mail: 1aialeksandrova@gmail.com 